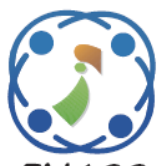

\title{
An Optimized Energy Efficient Routing for Wireless Sensor Network using Improved Spider Monkey Optimization Algorithm
}

\author{
Ravi Kumar Sanapala ${ }^{1 *}$ \\ Sreenivasa Rao Duggirala ${ }^{1}$ \\ ${ }^{1}$ Jawaharlal Nehru Technological University Hyderabad, \\ Department of Electronics and Communication Engineering, India \\ * Corresponding author's Email: ravi430us@ gmail.com
}

\begin{abstract}
Wireless Sensor Networks (WSNs) contain thousands of small and inexpensive sensor nodes distributed in the specific area for collecting the desired information from the environment. However, the sensor nodes have restricted battery energy, hence the random deployment of sensors creates issues while managing the resources for effective data delivery over the network. Therefore, this research aims to develop an energy-efficient cluster-based WSN to minimize the energy consumption of the nodes. In this paper, the Low-Energy Adaptive Clustering Hierarchy $(\mathrm{LEACH})$ algorithm is used for selecting the optimal Cluster Heads (CHs) from the network. Subsequently, the Improved Spider Monkey Optimization (ISMO) is used for creating the optimal routing through the CHs to the Base Station (BS) where the multiple fitness functions such as residual energy, distance, and routing traffic are considered to optimize the ISMO. Hence, the data communication between the intermediate CHs in hierarchical cluster-based architecture helps to minimize the energy consumption of the nodes. The performance of the proposed LEACH-ISMO method is analyzed using network lifetime, total energy consumption, normalized overhead, throughput, End-to-End Delay (EED) and Packet Delivery Ratio (PDR). The existing methods namely Fuzzy C-Means (FCM) with Artificial Bee Colony (ABC), Meta-Heuristic Ant Colony Optimization based Unequal Clustering (MHACO-UC) and Optimized Quality of Service-based Clustering and Multipath Routing Protocol (OQoS-CMRP) are used to evaluate the LEACH-ISMO method. The PDR of the LEACH-ISMO method is $96.7 \%$ for 200s, which is high when compared to the FCM-ABC, MHACO-UC and OQoS-CMRP.
\end{abstract}

Keywords: Cluster head, Energy consumption, Improved spider monkey optimization, Low-energy adaptive clustering hierarchy algorithm, Packet delivery ratio.

\section{Introduction}

Wireless sensor networks (WSNs) combine communication technology, distributed information processing technology, embedded computing technology, and sensor technology to accomplish wireless communications [1]. In general, the WSN is highly distributive and it is self-organized in its functionality which comprises the numerous tiny sensor nodes in the network. Since, the sensor node contains various components such as sensor, processor and communication unit [2-4]. The sensor nodes are battery-operated devices that observe the information from the environment and transmit it to the destination for performing an immediate action. Moreover, sensing, processing and communicating are the three main capacities of the sensors [5]. Each sensor node in the network observes the information from its nearby environment and transmits it to the important location namely base station (BS) or sink. Subsequently, the transmitted data is analyzed in the BS to achieve decision-making for diverse applications [6]. The sensors in the WSN sense various data such as pictures, video, temperature, humidity, pressure and so on [7]. Specifically, the WSN is used in an extensive range of applications in various fields such as military, agriculture and transportation $[8,9]$.

The power supply is considered an important part of the sensor node that is used to supply the required energy to the processors, sensors and transceiver. But, the restricted energy of the battery leads to the early exhaustion of network because of the extreme usage 
of the nodes. The energy efficiency is considered to be a challenging issue, due to the deployment of the sensors in the complex environment [10]. Clustering and routing are considered as two essential processes developed for energy efficiency of the WSN. The energy consumption of the nodes is minimized by using the clustering techniques in WSN. Accordingly, the sensors are separated into various groups called clusters, where a $\mathrm{CH}$ is selected from each cluster [11-13]. The CHs selected inside the cluster perform the critical operations such as data collection and transmission. However, the $\mathrm{CHs}$ forward the aggregated data packets to the BS on behalf of their own cluster members, hence there is a possibility of rapid energy depletion in the $\mathrm{CHs}$. This rapid energy exhaustion causes the routing interruption and network disconnection [14-16]. On the other hand, the communication task utilizes higher energy than the sensing and processing tasks. Therefore, it is essential to develop the energy efficient protocol for minimizing the energy consumption while transmitting the data packets [17]. The existing researches are used to accomplish the routing over the WSN are FCM with ABC [18], meta-heuristic ant colony optimization based unequal clustering (MHACO-UC) method [19], modified particle swarm optimization [20], firefly based routing [21], beesearch [22] and multi-constraints QoS routing [23]. The explanation of the aforementioned existing researches are given in the following sections.

Therefore, recent researchers aimed to improve the performance by adopting various protocols and algorithms. The main contributions of this research paper are given as follows: In this LEACH-ISMO method, the LEACH protocol is designed for finding direct gateway node (i.e., $\mathrm{CH}$ ) and the ISMO is designed on the optimized routing path detection states. With this LEACH-ISMO method, the overall performance is improved. The problem of this approach is that most of the WSNs are used in largescale applications. But, the sensors nodes having a restricted battery, so an effective routing method is developed in WSN. For providing an energy-efficient network, the LEACH-ISMO method is developed to accomplish an effective data transmission. A major objective of this approach is to improve the network performance by optimized routing method, which adopts the major parameters such as energy, distance and routing traffic for improving the performance is that energy and network lifetime.

This paper is organized as follows. Section 2 presents various literature related to this work. Section 3 presents the LEACH-ISMO method with algorithm novelty. Section 4 presents the results and its comparative analysis discussion. Section 5 concluded the LEACH-ISMO method with future scope.

\section{Literature survey}

Zongshan [18] presented the improved $\mathrm{ABC}$ to select the $\mathrm{CHs}$ from the network, since the optimal clustering was found by optimizing the FCM using the ABC algorithm. Subsequently, an enhanced version of the ant colony algorithm was developed to accomplish an energy-efficient routing algorithm. However, the developed ant-based routing algorithm considered only the energy-distance factor. Guleria and verma [19] presented the meta-heuristic ant colony optimization based unequal clustering (MHACO-UC) to perform an effective $\mathrm{CH}$ and transmission path selection. The principle of rendezvous node was developed in the unequal clustering for enhancing the lifetime of $\mathrm{CH}$. However, the ACO used in the WSN found the shortest path for transmission which failed to consider the energy of the nodes.

Deepa and suguna [20] developed the optimized QoS-based clustering and multipath routing protocol (OQoS-CMRP) to minimize energy consumption. The multi-hop communication path was found by using the singlesink-AllDestination algorithm. However, the OQoS-CMRP method failed to perform the fault tolerance during the communication. Giva andriana mutiara [21] presented the firefly synchronization multi-hop - geographical energyaware routing (FSM-GEAR) method was utilized to improve the energy efficiency by minimizing the transmission distance. Moreover, the waiting time was reduced by conducting the firefly synchronization among nodes. However, the weight was calculated only by the distance, energy and power consumption. For a better routing path generation, the routing traffic also required to be considered for minimizing the packet loss.

Mann and singh [22] presented the WSN routing strategy using the swarm intelligence approach. The assessment of reproduction results shows that beeswarm performs better as far as packet transmission, energy utilization, throughput and high network lifetime when compared to the other swarm intelligence based progressive routing protocols. However, the selection of $\mathrm{CH}$ considered only the energy and distance in the beeswarm.

Mostafaei [23] developed the multi-constraints QoS routing of WSN. QoS routing is one of the basic difficulties in WSNs, particularly for observation frameworks. To improve the proficiency of the organization with multi constraints QoS boundaries, a distributed learning robot based calculation was 
developed in WSN. However, this QoS-based routing doesn't analyze in the large-scale environment.

\section{LEACH-ISMO method}

Low energy adaptive clustering hierarchy (LEACH) is used on the cluster head selection process in WSN, which also utilizes the gateway discovery scheme with optimal routing strategy, which utilizes the spider monkey optimization algorithm. LEACH protocol utilizes a two-layer leveled structure with task randomization to similarly adjust the hubs. The technique can be applied for the plan of a few kinds of sensor networks that requires quality, energy productivity, versatility, network lifetime, and lesser transmission delay. The greater part of the LEACH variation conventions is intended to limit energy utilization since sensor hubs are energy imperatives. QoS relates to a few WSN execution issues like delay, data transmission, throughput and lifetime of the network. Fig. 1 shows the flowchart of the LEACH-ISMO method.

The wireless sensor networks have a base station and sensor hubs. The communication is carried out in two ways that are single and multi-hop communication. The $\mathrm{CH}$ uses the single-hop communication when the BS is in the range of source $\mathrm{CH}$. If the $\mathrm{BS}$ is located outside the transmission range, then the multi-hop transmission is accomplished using ISMO. Therefore, the combination of both the single and multi-hop data transmission improves energy efficiency. In the greater part of the WSN conventions; energy effectiveness is viewed as a key plan issue to improve the organization's lifetime.

\subsection{Gateway discovery scheme}

The gateway discovery scheme is used on the routing path selection of WSN. The $\mathrm{CH}$ selection, optimal route selection, and data transmission are performed effectively with QoS improvement. Here the threshold adopted greedy selection based SMO algorithm and gateway discovery scheme, are used with the LEACH protocol. Here the proposed gateway-based model is used to improve the lifetime of the network based on random $\mathrm{CH}$ selection using LEACH and appropriate route selection using ISMO. They have conveyed sensor hubs in the coherent locales based on their area in the detecting field. They have set the gate hub at the center of the detecting field. The entryway hub gathers information from different hubs in the field and sends it to the base station (BS).

Additionally, there is no requirement on the

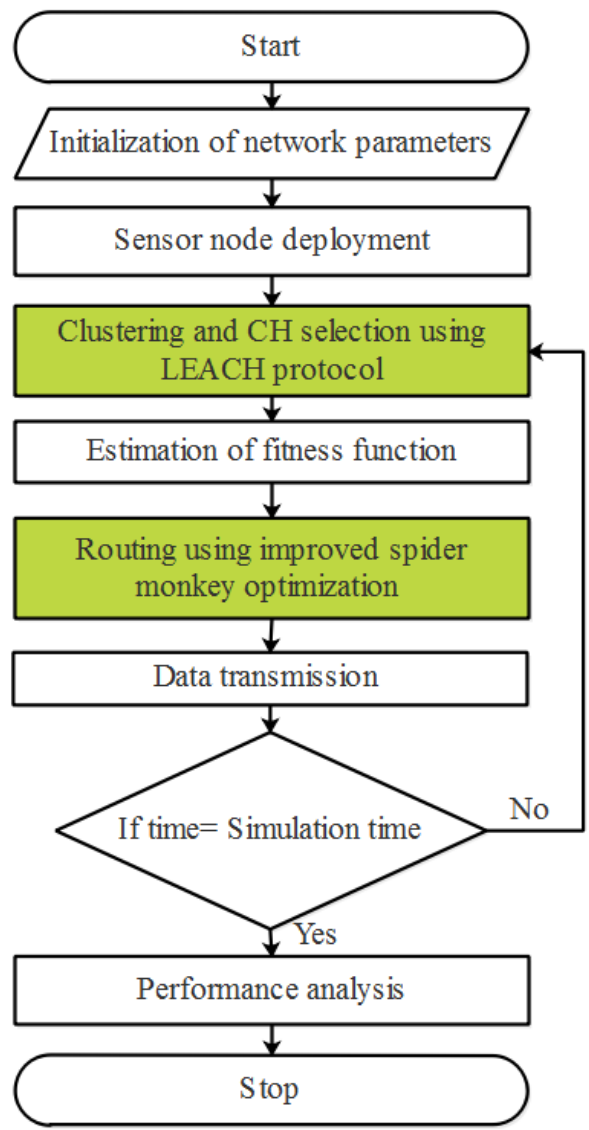

Figure. 1 Flowchart of LEACH-ISMO method

number of passage hubs, which can be sent in the overall network region. The LEACH approach includes the development of a cluster of sensor hubs fixated on the got signal quality and the utilization of a nearby $\mathrm{CH}$ as a switch to the BS. The decrease in energy utilization in information transmission is accomplished since the $\mathrm{CH}$ is associated with transmission to the BS instead of individual sensor hubs. LEACH is generally one of the hierarchical clustering protocols which is used to create a cluster using the signal strength and to select an optimal $\mathrm{CH}$ for aggregating the information from its cluster members. The CHs selected by using the LEACH are randomly changed for balancing the energy consumption of the nodes. If the node becomes a $\mathrm{CH}$ in a network, it cannot be a $\mathrm{CH}$ again for $P$ rounds. The random number generated in the range of $[0,1]$ and threshold value $(T)$ is calculated by using Eq. (1). This threshold value is used to select the $\mathrm{CH}$.

$$
T(n)= \begin{cases}\frac{P}{1-P \cdot\left(\operatorname{r.mod} \cdot\left(\frac{1}{P}\right)\right)} & \text { for } n \in G, \\ 0 & \text { Otherwise }\end{cases}
$$

Where, the required $\mathrm{CH}$ percentage is $P$ (e.g., $P=0.05)$; the number of nodes in the network is $n$, the group of nodes that haven't been selected as $\mathrm{CH}$ 
is represented as $G$ and the number of rounds are represented as $r$. Hence, the optimal node is chosen as $\mathrm{CH}$ based on the threshold value.

\subsection{Improved spider monkey optimization for data transmission}

Optimization techniques are utilized for discovering the ideal node of groups and choosing ideal quantities of node (optimal selection) in completely proposed LEACH replacements. Streamlining can be utilized for the ideal situation of sensor hubs and directing the way from hubs to the BS.

\subsubsection{Overview of SMO}

SMO is generally a population-based algorithm that is inspired by the social behavior of spider monkeys. This SMO mainly depends on the activities of spider monkeys which imitates the fission-fusion social structure. Moreover, SMO comprises of six iterative phases which are explained as follows:

\section{a. Local leader phase}

Initially, the current swarm is updated in this local leader phase. A fresh trial location is generated for each spider monkey by using a current location, location of the local leader and a randomly chosen member of the group are used to accomplish the local leader phase. The perturbation rate value is used to update each dimension of the solution. The

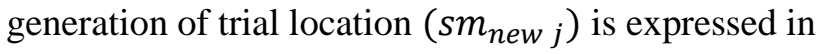
the Eq. (2).

$$
\begin{gathered}
s m_{n e w j}= \\
s m_{i j}+R(0,1) \times\left(l l_{k j}-s m_{i j}\right)+ \\
R(-1,1) \times\left(s m_{i j}-s m_{i j}\right) \text { if } R(0,1) \geq p r \\
s m_{i j} \text { Otherwise }
\end{gathered}
$$

Where, location of $i^{\text {th }}$ spider monkey for the coordinate $j$ is represented as $s m_{i j} ; R(0,1)$ and $R(-1,1)$ are the uniform random numbers between the $0 \& 1$, and $-1 \& 1$; location of $k^{\text {th }}$ local leader group for the coordinate $j$ is represented as $l l_{k j}$ and the perturbation rate is defined as $p r$. The new location is taken, when the generated location achieves improved fitness than the current location.

\section{b. Global leader phase}

This global leader phase updates only one solution which is randomly chosen from the entire population. The chosen spider monkey has the chance to update based on its probability. Eq. (3) shows the probability computation for the spider monkey $i$. Here, the fitness is calculated based on the distance between the individuals and their food source.

$$
\text { Probability }=0.9 \times\left(\frac{\text { fitness }_{i}}{\text { MaxFitness }}\right)+0.1
$$

Eq. (4) shows the generation of trail location for the spider monkey.

$$
\begin{aligned}
& s m_{n e w j}=s m_{i j}+R(0,1) \times\left(g l_{j}-s m_{i j}\right)+ \\
& R(-1,1) \times\left(s m_{r j}-s m_{i j}\right)
\end{aligned}
$$

Where, the $i^{\text {th }}$ spider monkey's fitness and fitness for the entire population are represented as fitness $_{i}$ and MaxFitness respectively; location of current global leader group for the coordinate $j$ is represented as $g l_{j}$; location of random chosen $r^{\text {th }}$ member for the coordinate $j$ is represented as $s m_{r j}$. Furthermore, the fitness of generated location and current location are compared to adopt a better solution.

\section{c. Global and local leader learning phase}

In global leader learning, the spider monkey which has the best fitness value is updated as a global leader. Similarly, the greedy selection is used in the local leader learning to choose the local leaders for each group.

\section{d. Local and global leader decision phase}

In the local leader decision phase, the random initialization or integration of global and local leaders are considering for updating the location of local leaders up to the predefined threshold namely local leader limit. On the other hand, global leaders are separated into small groups, when it is not updated up to predefined iterations namely global leader limit. Subsequently, the population is separated until achieving the maximum groups and the local leader learning is utilized for selecting the leader from the generated groups. Next, all the single groups are combined to generate the single group, when the location of a global leader is not updated and maximum groups are generated in the SMO.

\subsubsection{Routing path identification using ISMO}

In ISMO, the subgroups are integrated into a single group, when the position is not updated by the global optimal value. This process is utilized for balancing the exploitation and exploration capacity while maintaining the convergence speed. Therefore, the ISMO is tried to balance the population diversity 
by using three distinct fitness parameters energy, distance, routing traffic which leads to improving the route selection process in the WSN. Population initialization of SMO depends on the routing area and sensor utility. Then, local leader and global leader considerations are performed for grouping clusters. Here the LLP uses a greedy selection process with threshold adjustment logic and this can adjust the location of clusters by a fitness function. The GLP modify their position based on the higher cluster head value. In this, the greedy selection process helps to find the optimal solution of $\mathrm{CHs}$ and it is updated with current $\mathrm{CHs}$ position. Then it is labeled with new position and the threshold limit count of global leader is acted to update the new position of $\mathrm{CH}$. With the help of local leader decision and global leader decision, the $\mathrm{CH}$ is selected on end criteria of 100th iteration. The flow chart of SMO approach in $\mathrm{CHs}$ routing is shown in Fig. 2.

\section{$\mathrm{CH}$ selection in ISMO procedure:}

- Routing structure of clusters.

- Formation of cluster heads.

$$
\mathrm{CH}=\operatorname{Prob}_{i t} \times \mathrm{TCH}
$$

Where, Prob $_{i t}$ denotes probability of each iteration in clusters and $\mathrm{TCH}$ represents the total cluster heads used on the network with maximum iteration level.

- Measure the energy of sensor nodes ' $E_{n}$ ' and distance of each sensor nodes ' $D_{n}$ ' to the base station.

Distance evaluation is given by,

$$
D_{n}=\sqrt{(a)^{2}+(b)^{2}}
$$

Where, $a$ and $b$ are the active sensor nodes

- Update the energy requirement of cluster head with higher-level priority.

- Data aggregation within the cluster's information.

- Data transmission based on nearest cluster heads to higher-level $\mathrm{CH} / \mathrm{BS}$.

- Maximum energy updated cluster heads by updating fitness value.

$$
\text { fitness }(n)=\propto E_{n}+\beta \frac{1}{T r_{n}}+\gamma \frac{1}{D_{n}}
$$

Where, $E_{n}$ represents the energy, $D_{n}$ represents the distance, $r_{n}$ represents routing traffic node $T$ with a number of nodes $n$ and $\alpha, \beta, \gamma$ are the weighted coefficients. Better QoS performance is obtained

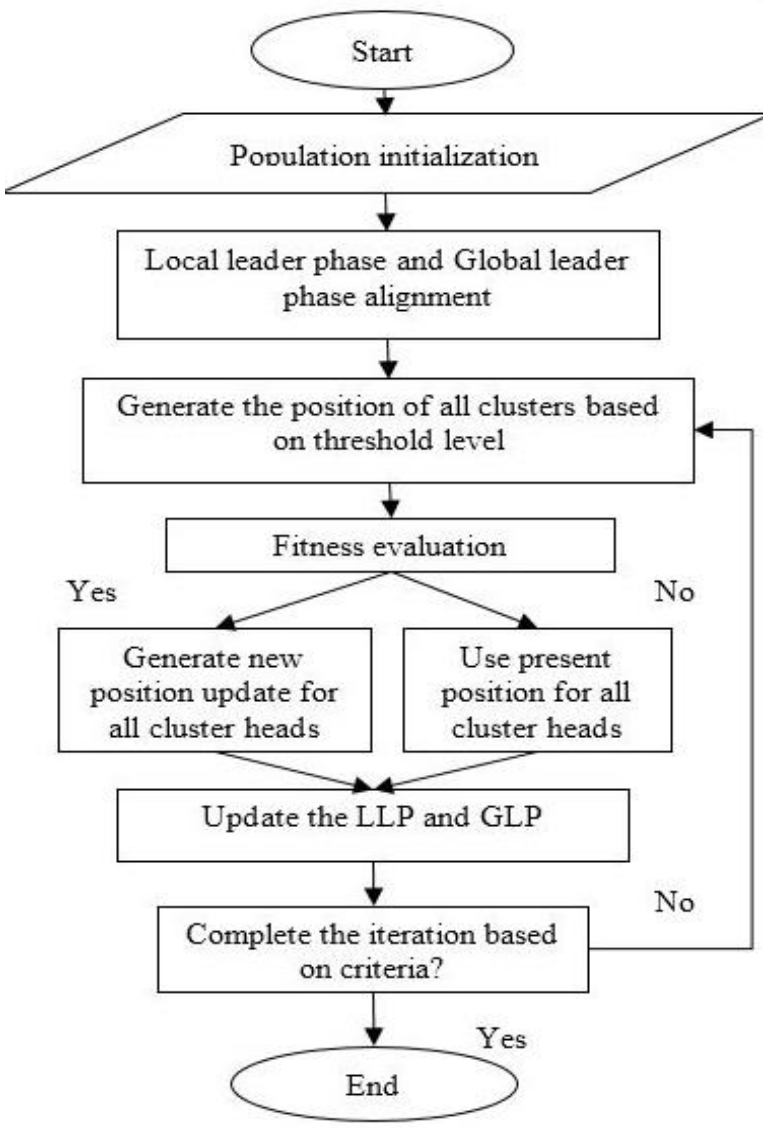

Figure. 2 Flow chart of SMO approach in $\mathrm{CHs}$ routing

based on energy and network lifetime. Probability evaluation of LLP and GLP is obtained by using Eq. (7) in Eq. (3).

After cluster development, $\mathrm{CHs}$ are assigned that are used as an innovator in each group. $\mathrm{CHs}$ are burdened with the obligation regarding information accumulation and performing routing for its group part's data to the base station. Likewise, the groups that comprise of multi-hubs have a higher weight than bunches with less hubs as the $\mathrm{CHs}$ for those enormous estimated groups need to get, total and communicate more information.

A CH can be chosen arbitrarily or pre-allotted by the architect of the organization. A CH can likewise be chosen by contemplating the remaining energy of hubs in the group. The $\mathrm{CHs}$ are known to have higher weights than part hubs; along these lines, the job of $\mathrm{CH}$ is changed to share the weight and consequently improve the lifetime of those clusters. The energy considered in the ISMO is used to avoid the node/link failure while transmitting the data packets. Subsequently, the energy consumption of the node is minimized based on the distance and energy used in the fitness function. Specifically, the shortest path identification of ISMO results in lesser energy consumption which leads to an increase the energy efficiency of the WSN. 


\section{Results and discussion}

The results and discussion of the LEACH-ISMO method is clearly explained in this section. The implementation and simulation of the LEACH-ISMO method is done in the Network simulator-2.34 where the system functions with 4-GB RAM and Intel core processor. The simulation parameters of the LEACHISMO method are mentioned in Table 1.

The performance of the LEACH-ISMO method is analyzed by means of network lifetime, total energy consumption, normalized overhead, throughput, EED and PDR. The existing methods such as FCM-ABC, OQoS-CMRP and MHACO-UC methods are used to evaluate the performance of the LEACH-ISMO method, where the existing methods are implemented and simulated by using the same specifications mentioned in Table 1.

\subsection{Network lifetime}

Network lifetime generally depends on the alive

Table 1. Simulation parameters

\begin{tabular}{|c|c|}
\hline Parameters & Values \\
\hline Area & $300 \mathrm{~m} \times 200 \mathrm{~m}$ \\
\hline Number of nodes & $50,100,150,200 \& 250$ \\
\hline MAC Type & IEEE 802.15.4 \\
\hline Radio Propagation Model & $\begin{array}{c}\text { Two-ray ground } \\
\text { reflection model }\end{array}$ \\
\hline Queue Type & Priority Queue \\
\hline Channel Type & Channel/wireless \\
\hline Antenna Type & Omnidirectional \\
\hline Traffic source & CBR \\
\hline Data Packet size & 128 bytes \\
\hline Control Packet size & 32 bytes \\
\hline The initial energy of nodes & 3 Joules \\
\hline Simulation time & $500 \mathrm{~s}$ \\
\hline
\end{tabular}

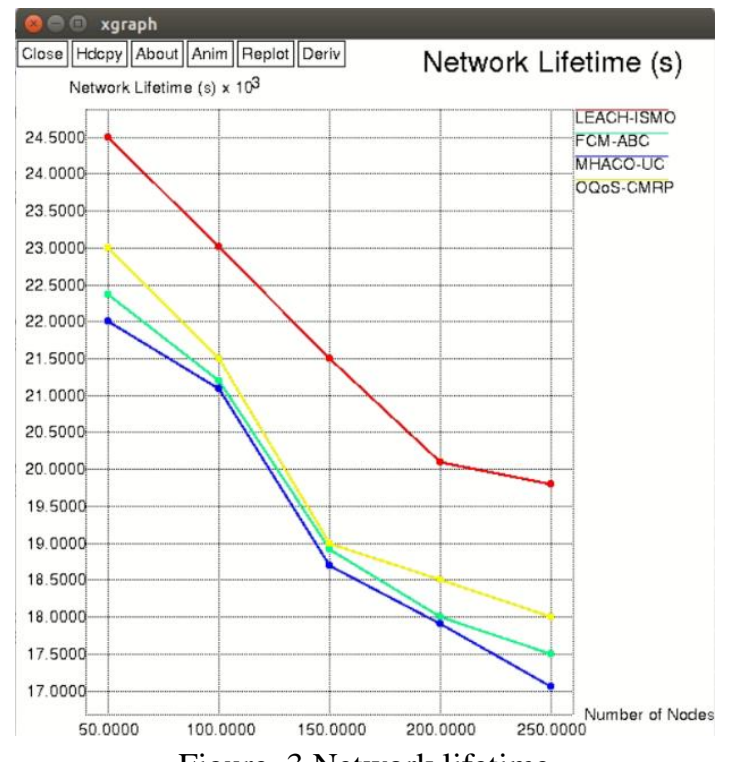

Figure. 3 Network lifetime nodes and connectivity that exist in the network, hence the energy consumption should be minimized for achieving a better lifetime.

Fig. 3 shows the network lifetime comparison of the FCM-ABC [18], MHACO-UC [19], OQoSCMRP [20] and LEACH-ISMO method. Fig. 3 clearly shows that the LEACH-ISMO method achieves a higher lifetime than the FCM-ABC [18], MHACO-UC [19] and OQoS-CMRP [20]. The network lifetime of the LEACH-ISMO method is increased, because of its random $\mathrm{CH}$ selection and shortest path identification during the communication. Since, the transmission path with lesser distance leads to obtain less energy consumption which helps to increase the network lifetime.

\subsection{Total energy consumption}

The sensor's energy consumption is the difference among its initial energy and residual energy.

The comparison of energy consumption for the LEACH-ISMO method with FCM-ABC [18], MHACO-UC [19] and OQoS-CMRP [20] are shown in Fig. 4. The energy consumption of the LEACHISMO method is less when compared to the FCMABC [18], MHACO-UC [19] and OQoS-CMRP [20]. Due to the shortest path identification of the ISMO, the energy consumption of the nodes is minimized while transmitting the data packets.

\subsection{Normalized overhead}

Normalized overhead is the ratio between the amount of forwarded control packets and the amount of data packets received by the BS.

Fig. 5 shows the normalized overhead

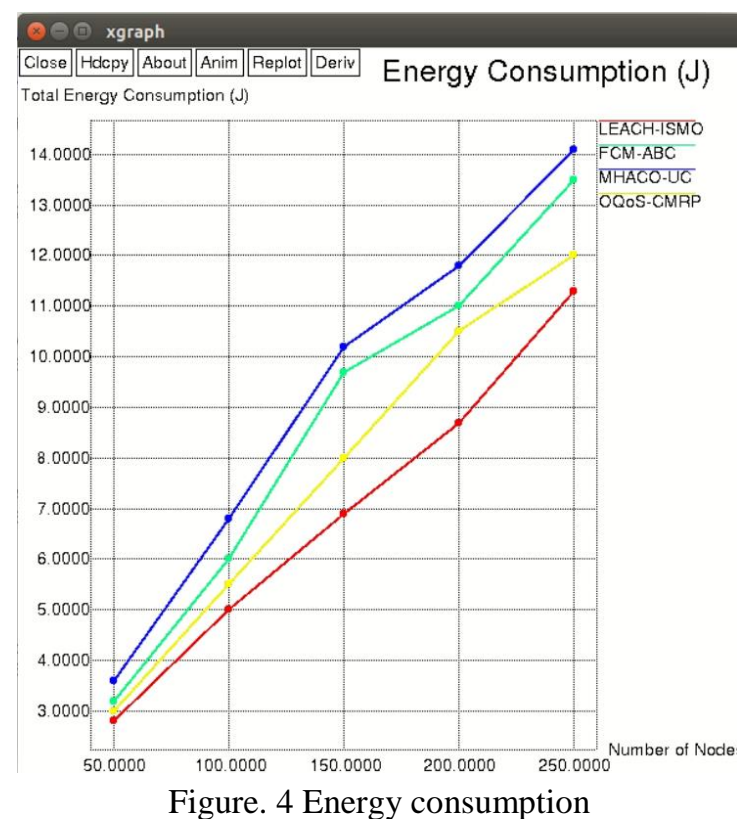

Figure. 4 Energy consumption 


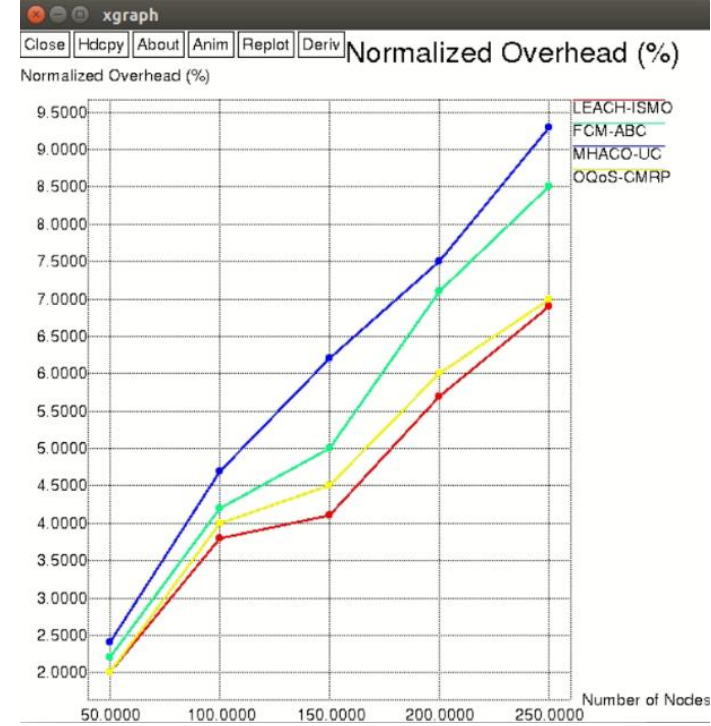

Figure. 5 Normalized overhead

comparison of the FCM-ABC [18], MHACO-UC [19], OQoS-CMRP [20] and LEACH-ISMO method. Fig. 5 clearly shows that the LEACH-ISMO method achieves less normalized overhead than the FCMABC [18], MHACO-UC [19] and OQoS-CMRP [20]. The FCM-ABC [18], MHACO-UC [19] and OQoSCMRP [20] achieves higher control overhead because it broadcasts high amount of control picketer while generating the data transmission path. But, the LEACH-ISMO requires only less amount of control packets due to the fitness function considered in the ISMO.

\subsection{Throughput}

Throughput is defined as the number of packets successfully received at the BS in certain time.

The comparison of throughput for the LEACHISMO method with FCM-ABC [18], MHACO-UC [19] and OQoS-CMRP [20] are shown in Fig. 6. The throughput of the LEACH-ISMO method is high when compared to the FCM-ABC [18], MHACO-UC [19] and OQoS-CMRP [20]. The throughput of the LEACH-ISMO method is increased by minimizing the energy consumption of the nodes. Because, if the energy is exhausted in the transmitting node, the packet loss occurs in the WSN. On the other hand, the energy considered in the ISMO's fitness function helps to avoid node failure in the routing path. Therefore, the throughput of the LEACH-ISMO method is increased by avoiding node failure.

\subsection{End to end delay}

EED is calculated as the total amount of time utilized by the data packet to be forwarded from the source node to the BS.

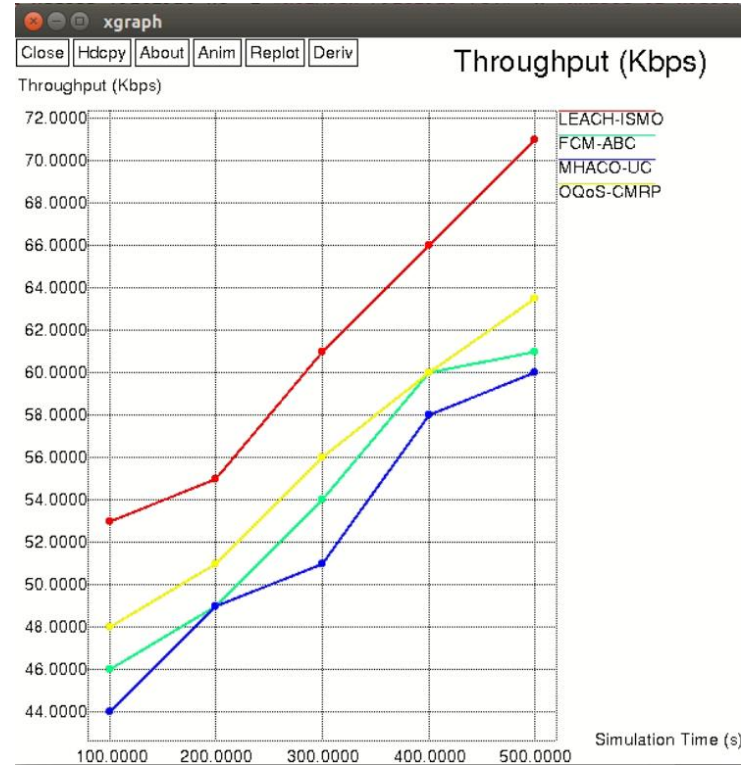

Figure. 6 Throughput

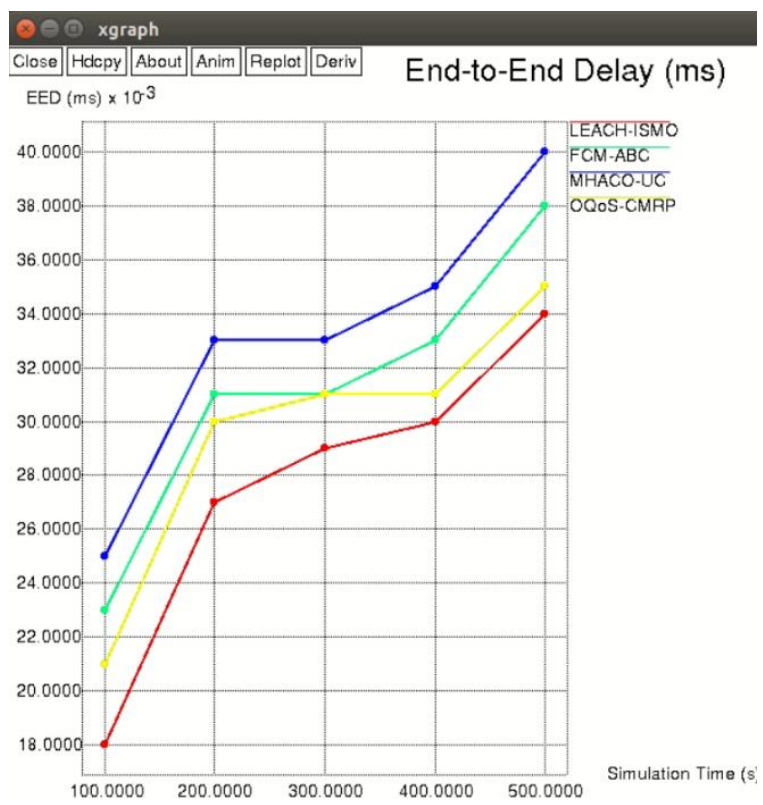

Figure. 7 End to end delay

Fig. 7 shows the EED comparison of the FCMABC [18], MHACO-UC [19], OQoS-CMRP [20] and LEACH-ISMO methods. Fig. 7 clearly shows that the LEACH-ISMO method achieves lesser EED than the FCM-ABC [18], MHACO-UC [19] and OQoS-CMRP [20]. The EED of the LEACH-ISMO method is reduced because of the less processing delay and less transmission delay achieved by the shortest path identification of ISMO.

\subsection{Packet delivery ratio}

PDR is generally the ratio between the amount of data packets received by the BS and the amount of data packets forwarded by the source node.

The comparison of PDR for the LEACH-ISMO 
Table 2. Comparative analysis of network lifetime, total energy consumption and normalized overhead for LEACHISMO in terms of number of nodes

\begin{tabular}{|c|c|c|c|c|c|c|}
\hline \multirow{2}{*}{ Performances } & Methods & \multicolumn{4}{|c|}{ Number of nodes } \\
\cline { 2 - 6 } & & $\mathbf{5 0}$ & $\mathbf{1 0 0}$ & $\mathbf{1 5 0}$ & $\mathbf{2 0 0}$ & $\mathbf{2 5 0}$ \\
\hline \multirow{3}{*}{ Network lifetime (s) } & FCM-ABC [18] & 22360 & 21200 & 18920 & 18000 & 17500 \\
\cline { 2 - 6 } & MHACO-UC [19] & 22000 & 21090 & 18690 & 17900 & 17050 \\
\cline { 2 - 6 } & OQoS-CMRP [20] & 23000 & 21500 & 19000 & 18500 & 18000 \\
\cline { 2 - 6 } & LEACH-ISMO & 24500 & 23010 & 21500 & 20100 & 19800 \\
\hline \multirow{3}{*}{ Total energy consumption (J) } & FCM-ABC [18] & 3.2 & 6 & 9.7 & 11 & 13.5 \\
\cline { 2 - 6 } & MHACO-UC [19] & 3.6 & 6.8 & 10.2 & 11.8 & 14.1 \\
\cline { 2 - 6 } & OQoS-CMRP [20] & 3 & 5.5 & 8 & 10.5 & 12 \\
\cline { 2 - 6 } & LEACH-ISMO & 2.8 & 5.0 & 6.9 & 8.7 & 11.3 \\
\hline \multirow{3}{*}{ Normalized overhead (\%) } & FCM-ABC [18] & 2.2 & 4.2 & 5 & 7.1 & 8.5 \\
\cline { 2 - 6 } & MHACO-UC [19] & 2.4 & 4.7 & 6.2 & 7.5 & 9.3 \\
\cline { 2 - 6 } & OQDS-CMRP [20] & 2 & 4 & 4.5 & 6 & 7 \\
\cline { 2 - 6 } & LEACH-ISMO & 2 & 3.8 & 4.1 & 5.7 & 6.9 \\
\hline
\end{tabular}

Table 3. Comparative analysis of throughput, EED and PDR for LEACH-ISMO in terms of simulation time

\begin{tabular}{|c|c|c|c|c|c|c|}
\hline \multirow{2}{*}{ Performances } & Methods & \multicolumn{4}{|c|}{ Simulation time (s) } \\
\cline { 2 - 6 } & & $\mathbf{1 0 0}$ & $\mathbf{2 0 0}$ & $\mathbf{3 0 0}$ & $\mathbf{4 0 0}$ & $\mathbf{5 0 0}$ \\
\hline \multirow{3}{*}{ Throughput (Kbps) } & FCM-ABC [18] & 46 & 49 & 54 & 60 & 61 \\
\cline { 2 - 7 } & MHACO-UC [19] & 44 & 49 & 51 & 58 & 60 \\
\cline { 2 - 7 } & OQoS-CMRP [20] & 48 & 51 & 56 & 60 & 63.5 \\
\cline { 2 - 7 } & LEACH-ISMO & 53 & 55 & 61 & 66 & 71 \\
\hline \multirow{3}{*}{ EED (ms) } & FCM-ABC [18] & 0.023 & 0.031 & 0.031 & 0.033 & 0.038 \\
\cline { 2 - 7 } & MHACO-UC [19] & 0.025 & 0.033 & 0.033 & 0.035 & 0.04 \\
\cline { 2 - 7 } & OQoS-CMRP [20] & 0.021 & 0.03 & 0.031 & 0.031 & 0.035 \\
\cline { 2 - 7 } & LEACH-ISMO & 0.018 & 0.027 & 0.029 & 0.03 & 0.034 \\
\hline \multirow{3}{*}{ PDR (\%) } & FCM-ABC [18] & 94.3 & 92 & 90.5 & 90 & 85 \\
\cline { 2 - 7 } & MHACO-UC [19] & 93 & 91.2 & 88 & 87.3 & 85 \\
\cline { 2 - 7 } & OQoS-CMRP [20] & 95 & 93 & 92 & 89 & 87 \\
\cline { 2 - 7 } & LEACH-ISMO & 98.9 & 96.7 & 95.2 & 94.3 & 93.0 \\
\hline
\end{tabular}

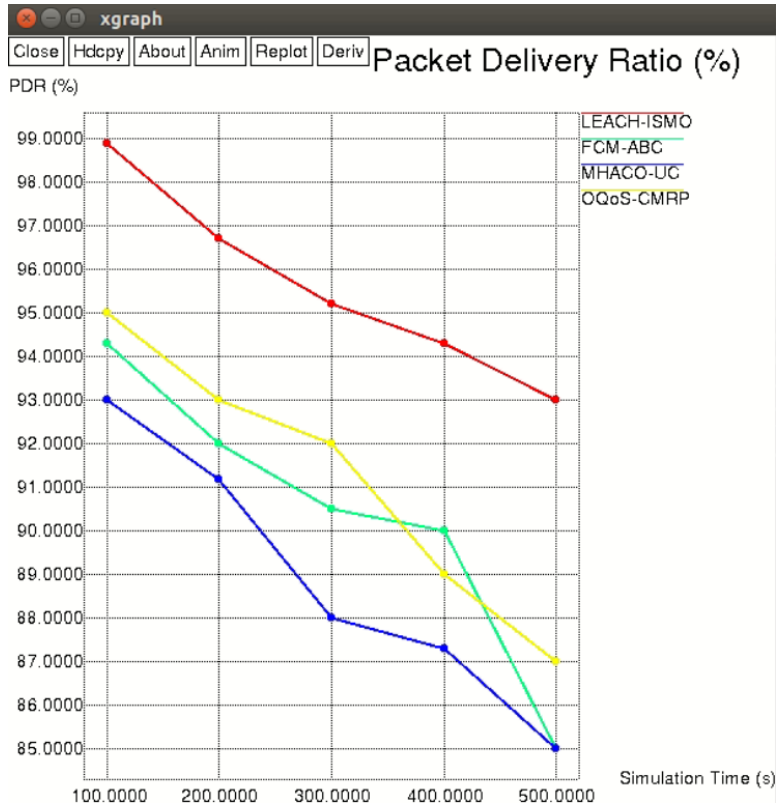

Figure. 8 Packet delivery ratio

method with FCM-ABC [18], MHACO-UC [19] and OQoS-CMRP [20] are shown in Fig. 8. The PDR of the LEACH-ISMO method is high when compared to the FCM-ABC [18], MHACO-UC [19] and OQoSCMRP [20]. The higher network lifetime of LEACHISMO method helps to increase the PDR of the network. Further, the packet loss is minimized by avoiding the node failure by considering the residual energy of $\mathrm{CH}$ in the ISMO's fitness function.

Tables 2 and 3 show the comparative analysis of the LEACH-ISMO method in terms of number of nodes and simulation time respectively. From Tables 2 and 3, it is concluded that the LEACH-ISMO method provides better performance than the MHACO-UC [19] and OQoS-CMRP [20]. For example, the PDR of the LEACH-ISMO method is $96.7 \%$ for 200 s which is high when compared to the FCM-ABC [18], MHACO-UC [19] and OQoSCMRP [20]. The existing methods MHACO-UC [19] and OQoS-CMRP [20] obtains less performance because it failed to avoid the node failure/ link failure during the data transmission. Additionally, the FCM$\mathrm{ABC}[18]$ failed to consider the routing traffic during routing path generation. The packet loss of the network is high, when there is high traffic between 
the nodes. However, the LEACH-ISMO method avoids node failure by considering the residual energy of the node during the routing path generation which helps to improve the packet delivery over the network. The route traffic consideration of LEACHISMO leads to minimize the packet loss over the network. Subsequently, the shortest path identification using the ISMO leads to minimizing the energy consumption of the nodes, and thus results in a higher network lifetime. Meanwhile, the appropriate fitness function of ISMO doesn't require a high amount of control packets during the route discovery process, hence it used to minimize the control overhead of the LEACH-ISMO method.

\section{Conclusion}

In this paper, the gateway discovery is done by using LEACH and routing is done by using ISMO to improve the performances of WSN. Here the spider monkey optimization algorithm is applied to the routing network for finding optimal cluster heads. Quality of service is improved for major parameters of WSN which are energy consumption, network lifetime, and throughput. The comparison results show that the LEACH-ISMO method achieves better performance than existing FCM-ABC, MHACO-UC and OQoS-CMRP. The routing of WSN analyses the performance of WSN parameters such as energy consumption, throughput, packet delivery rate, packet loss, end-to-end delay and network lifetime. The PDR of the LEACH-ISMO method is $96.7 \%$ for 200s, which is high when compared to the FCM-ABC, MHACO-UC and OQoS-CMRP. In the future, an adaptive path can be selected by using the hybrid optimization algorithm for improving the overall performances.

\section{Conflicts of interest}

The authors declare no conflict of interest.

\section{Author contributions}

The paper background work, conceptualization, methodology, dataset collection, implementation, result analysis and comparison, preparing and editing draft, visualization have been done by first author. The supervision, review of work and project administration, has been done by second author.

\section{References}

[1] T. Wang, G. Zhang, X. Yang, and A. Vajdi, "Genetic Algorithm for Energy-Efficient Clustering and Routing in Wireless Sensor
Networks", Journal of Systems and Software, Vol. 146, pp. 196-214, 2018.

[2] K. Muthukumaran, K. Chitra, and C. Selvakumar, "An Energy Efficient Clustering Scheme Using Multilevel Routing for Wireless Sensor Network", Computers \& Electrical Engineering, Vol. 69, pp. 642-652, 2018.

[3] S. M. Bozorgi and A. M. Bidgoli, "HEEC: A Hybrid Unequal Energy Efficient Clustering for Wireless Sensor Networks", Wireless Networks, Vol. 25, pp. 4751-4772, 2019.

[4] A. Panchal and R. K. Singh, "EHCR-FCM: Energy efficient hierarchical clustering and routing using fuzzy $\mathrm{C}$-means for wireless sensor networks", Telecommunication Systems, Vol. 76, No. 2, pp. 251-263, 2021.

[5] R. Sharma, V. Vashisht, and U. Singh, "EEFCMDE: Energy-Efficient Clustering based on Fuzzy $C$ Means and Differential Evolution Algorithm in WSNs", IET Communications, Vol. 13, No. 8, pp. 996-1007, 2019.

[6] P. S. Mann and S. Singh, "Artificial Bee Colony Metaheuristic for Energy-Efficient Clustering and Routing in Wireless Sensor Networks", Soft Computing, Vol. 21, pp. 6699-6712, 2017.

[7] S. P. Singh and S. Sharma, "Genetic-AlgorithmBased Energy-Efficient Clustering (GAEEC) for Homogenous Wireless Sensor Networks", IETE Journal of Research, Vol. 64, pp. 648-659, 2018.

[8] Z. Zhao, K. Xu, G. Hui, and L. Hu, "An EnergyEfficient Clustering Routing Protocol for Wireless Sensor Networks Based on AGNES with Balanced Energy Consumption Optimization", Sensors, Vol. 18, p. 3938, 2018.

[9] K. Xu, Z. Zhao, Y. Luo, G. Hui, and L. Hu, “An Energy-Efficient Clustering Routing Protocol based on a High-QoS Node Deployment with an Inter-Cluster Routing Mechanism in WSNs", Sensors, Vol. 19, No. 12, p. 2752, 2019.

[10] S. M. Palvinder and S. Satvir, "Energy Efficient Clustering Protocol based on Improved Metaheuristic in Wireless Sensor Networks", Journal of Network and Computer Applications, Vol. 83, pp. 40-52, 2017.

[11] S. K. Gupta and P. K. Jana, "Energy Efficient Clustering and Routing Algorithms for Wireless Sensor Networks: GA Based Approach", Wireless Personal Communications, Vol. 83, pp. 2403-2423, 2015.

[12] P. Nayak and B. Vathasavai, "Energy Efficient Clustering Algorithm for Multi-Hop Wireless Sensor Network Using Type-2 Fuzzy Logic", IEEE Sensors Journal, Vol. 17, No. 14, pp. 44924499, 2017. 
[13] D. I. Reddy, C. Puttamadappa, and H. N. Suresh, "Merged glowworm swarm with ant colony optimization for energy efficient clustering and routing in Wireless Sensor Network", Pervasive and Mobile Computing, Vol. 71, p. 101338, 2021.

[14] A. Rodríguez, C. D. V. Soto, and R. Velázquez, "Energy-Efficient Clustering Routing Protocol for Wireless Sensor Networks based on Yellow Saddle Goatfish Algorithm", Mathematics, Vol. 8, No. 9, p. 1515, 2020.

[15] N. Mittal, U. Singh, and B. S. Sohi, "A Stable Energy Efficient Clustering Protocol for Wireless Sensor Networks", Wireless Networks, Vol. 23, No. 6, pp. 1809-1821, 2017.

[16] K. Haseeb, B. K. Abu, A. Ahmed, T. Darwish, and I. Ahmed, "WECRR: Weighted EnergyEfficient Clustering with Robust Routing for Wireless Sensor Networks", Wireless Personal Communications, Vol. 97, No. 1, pp. 695-721, 2017.

[17] N. Morsy, E. Abdelhay, and S. Kishk, "Proposed Energy Efficient Algorithm for Clustering and Routing in WSN", Wireless Personal Communications, Vol. 103, No. 3, pp. 2575-2598, 2018.

[18] W. Zongshan, D. Hongwei, L. Bo, B. Liyong, and Y. Zhijun, "An Energy Efficient Routing Protocol Based on Improved Artificial Bee Colony Algorithm for Wireless Sensor Networks", IEEE Access, Vol. 8, pp. 133577133596, 2020.

[19] K. Guleria and A. K. Verma, "Meta-heuristic Ant Colony Optimization Based Unequal Clustering for Wireless Sensor Network", Wireless Personal Communications, Vol. 105, pp. 891-911, 2019.

[20] O. Deepa and J. Suguna, "An Optimized QoSbased Clustering with Multipath Routing Protocol for Wireless Sensor Networks", Journal of King Saud University-Computer and Information Sciences, Vol. 32, No. 7, pp. 763-774, 2020.

[21] G. A. Mutiara, O. Mohd, N. Suryana, and A. N. Pee, "Weights-Based Energy-Efficient Wireless Sensor Network Protocol with Firefly Synchronization for Illegal Logging", International Journal of Intelligent Engineering and Systems, Vol. 14, No. 3, 2021.

[22] P. S. Mann and S. Singh, "Energy-Efficient Hierarchical Routing for Wireless Sensor Networks: A Swarm Intelligence Approach", Wireless Personal Communications, Vol. 92, No. 2, pp. 785-805, 2017.

[23] H. Mostafaei, "Energy-Efficient Algorithm for Reliable Routing of Wireless Sensor Networks",
IEEE Transactions on Industrial Electronics, Vol. 66, No. 7, pp. 5567-5575, 2019. 